\title{
Quantitative Specimen Electric Potential Maps Using Segmented and Pixel Detectors in Scanning Transmission Electron Microscopy
}

\author{
H.G. Brown ${ }^{1}$, N. Shibata ${ }^{2}$, Z. Chen ${ }^{3}$, M. Weyland ${ }^{1}$, T.C. Petersen ${ }^{1}$, D.M. Paganin ${ }^{1}$, M.J. Morgan ${ }^{1}$, \\ H. Sasaki ${ }^{4}$, L. J. Allen ${ }^{5}$ and S. D. Findlay ${ }^{1}$ \\ 1. School of Physics and Astronomy, Monash University, Melbourne, Australia \\ 2. Crystal Interface Laboratory, Institute of Engineering Innovation, University of Tokyo, Tokyo, Japan \\ 3. School of Applied and Engineering Physics, Cornell University, Ithaca, USA \\ 4. Furukawa Electric Ltd., Yokohama, Japan \\ 5. School of Physics, University of Melbourne, Melbourne, Australia
}

Research into materials for the next generation of computers, batteries and solar cells requires techniques that can characterise both the structural and functional properties of materials, often at atomic resolution. Over the past fifteen years or so, advances in scanning transmission electron microscopy (STEM) have led to a technique capable of atomic resolution imaging of the heavy and light atomic positions in a sample and elemental mapping. Recently, new developments in segmented and pixel detectors for STEM have made it possible to record more detailed information about the interaction of the electron probe with the specimen. This talk will discuss quantitative retrieval of specimen electric fields from segmented and pixel detector data. We explore two different case studies: atomic resolution imaging of monolayer $\mathrm{MoS}_{2}$ and thicker samples of the perovskite $\mathrm{SrTiO}_{3}$, and nanometre scale resolution imaging of the inbuilt electric field of a $p$ - $n$ junction in a GaAs semiconductor.

We discuss two approaches to studying the electric potential in STEM: segmented detector ptychography (SDP) [1] and differential phase contrast (DPC) [2,3]. In SDP, we solve a set of linear equations which relate the set of STEM images for three or more diffraction plane detectors to a specimen transmission function [1]. The phase of the transmission function is proportional to the specimen projected electric potential. In DPC STEM, we use the fact that the specimen electric field for the specimen region illuminated by the probe can be related to the first moment of the diffraction plane intensity (the diffraction plane "centre of mass") for that probe position. Both segmented detector ptychography and DPC assume that the object is much thinner than the probe depth of focus.

For imaging of a $\mathrm{MoS}_{2}$ monolayer it is possible to quantitatively reconstruct the specimen potential [see Fig. 1 (a)]. For the thicker $\mathrm{SrTiO}_{3}$ specimens both SDP and DPC significantly underestimate the projected electric potential [see Fig. 1 (b)] as a result of implicit assumptions that the object is thin (and weakly scattering in the case of SDP). A modification to the SDP algorithm to take into account the finite thickness of the object improves our reconstruction somewhat, but not to the extent that we can claim a quantitative reconstruction of the potential at atomic resolution for a thick object.

For nanometre scale imaging, the size of the probe forming aperture can be made smaller, making the depth of field considerably larger. This better validates the approximations made by SDP and DPC. As a test case, we use segmented detector STEM images of a $p-n$ junction in a GaAs specimen; examples of which are shown in Fig. 2 along with derived centre of mass images. We show that it is possible to quantitatively reconstruct the electric field using both algorithms. Careful calibration of the detector response to changes in the diffraction plane "centre of mass" are required and these reveal how inelastic 
scattering due to excitation of plasmons by the electron probe affects our measurement of the specimen electric field [4].

\section{References:}

[1] H.G. Brown et al, Phys. Rev. B 93 (2016), p. 134116.

[2] R. Close et al, Ultramicroscopy 159 (2015), p. 124.

[3] Z. Chen et al, Ultramicroscopy 169 (2016), p. 107.

[4] The authors acknowledge funding from the Australian Research Council's Discovery Projects funding scheme (Projects No. DP140102538 and DP160102338).
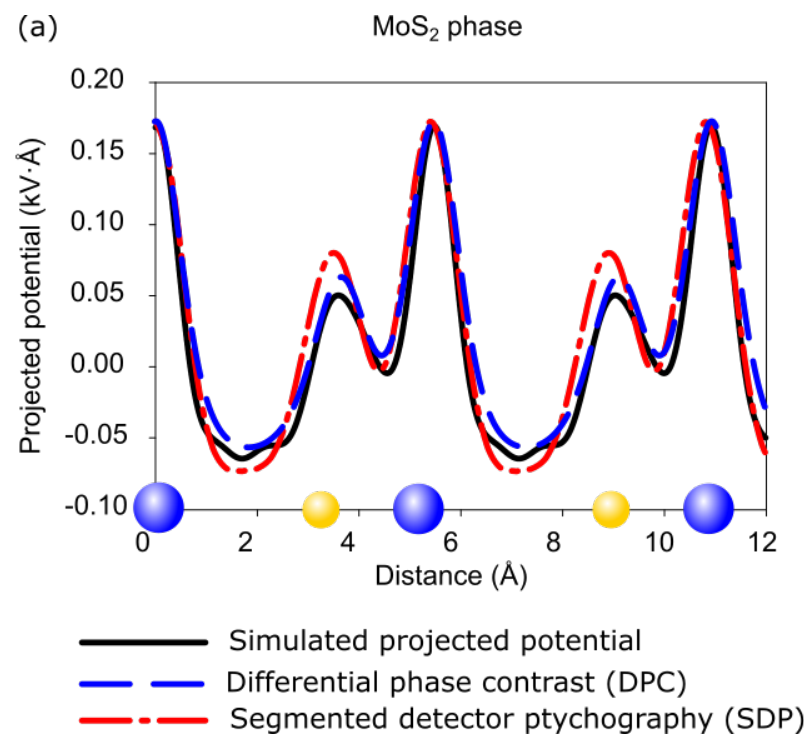
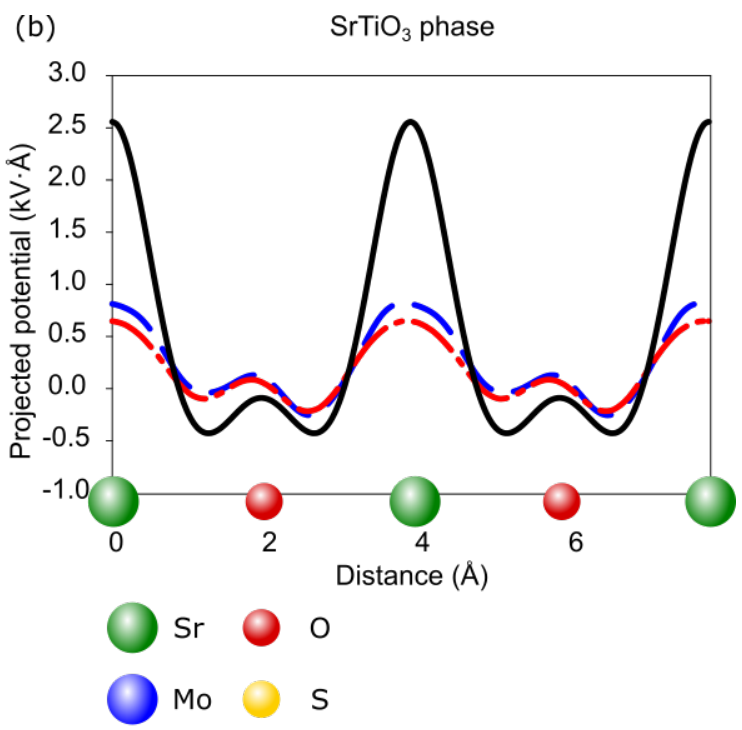

Figure 1. Retrieved projected potential for (a) monolayer $\mathrm{MoS}_{2}$ and (b) a $78.1 \AA$ thick $\mathrm{SrTiO}_{3}$ sample. The projected potential for segmented detector ptychography (SDP) and differential phase contrast (DPC) show good agreement with each other and the simulated $\mathrm{MoS}_{2}$ potential. For thick $\mathrm{SrTiO}_{3}$ sample both SDP and DPC retrieve a similar phase but significantly underestimate the phase suggested through simulation.

(a) STEM images

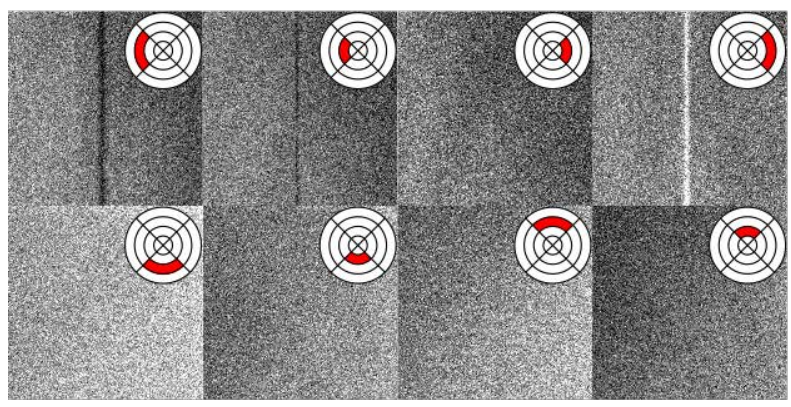

(b) Centre of mass images

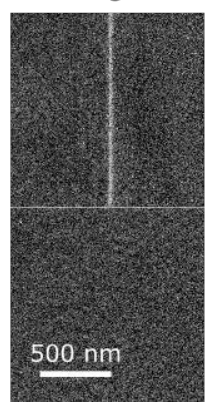

(c) Segmented detector set-up

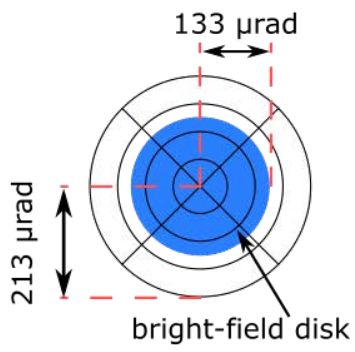

Figure 2. Electric field mapping of a $p-n$ junction in a GaAs sample using segmented detectors. The electric field is visible in (a) the STEM images for detector segments oriented along the horizontal direction (top row), whereas the STEM images oriented in the vertical direction (bottom row) show little contrast. (b) The horizontal and vertical components of the electric field map in the top and bottom rows in (a) respectively. (c) Schematic of the detector geometry. 